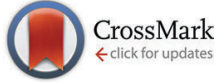

Cite this: Phys. Chem. Chem. Phys., 2015, 17, 9471

Received 18th December 2014, Accepted 2nd March 2015

DOI: $10.1039 / c 4 c p 05944 a$

www.rsc.org/pccp

\title{
Qualifying label components for effective biosensing using advanced high-throughput SEIRA methodology $\dagger$
}

\author{
Andrea Hornemann, ${ }^{* a}$ Diane Eichert, ${ }^{b}$ Sabine Flemig, ${ }^{c}$ Gerhard Ulm ${ }^{a}$ and \\ Burkhard Beckhoffa
}

\begin{abstract}
The need for technological progress in bio-diagnostic assays of high complexity requires both fundamental research and constructing efforts on nano-scaled assay recognition elements that can provide unique selectivity and design-enhanced sensitivity features. Nanoparticle induced sensitivity enhancement and its application related to multiplexed capability Surface-Enhanced InfraRed Absorption (SEIRA) assay formats are well suitable for these purposes. The potential of diverse fluorophore-antibody conjugates, being chemisorbed onto low-cost gold nanoparticulate SEIRA substrates, has been explored with respect to their spectral discriminability. These novel biolabels deliver molecular SEIRA fingerprints that have been successfully analyzed by both uni- and multivariate analyzing tools, to discriminate their multiplexing capabilities. We show that this robust spectral encoding via SEIRA fingerprints opens up new opportunities for a fast, reliable and multiplexed high-end screening in biodiagnostics.
\end{abstract}

\section{Introduction}

Nanotechnology has an increasing impact on both the design and functionality of biomedical and bio-diagnostic devices for reliable high-performance analysis. High-throughput capabilities require the simultaneous detection of various analytes combined with appropriate bioassay components. Additionally, reduction of the biosensors' cost by using, for instance, low cost nanoparticles (NPs) instead of artificial nanostructures is another important aspect. Various bioassay configurations may be used, such as the immobilization of targeting elements such as antibodies on glass slides coated with various targeting ligands, as microarrays. To design assays for antibodies that function as molecular probes, a selective binding event has to be optimized with respect to the overall physicochemical properties which may influence the successful readout of signals. This constitutes the core of the first design steps to elaborate multi-purpose bio-assay platforms. Bio-assays are standardized tests usually implemented to support multiple intended uses such as the detection of

\footnotetext{
${ }^{a}$ Physikalisch-Technische Bundesanstalt, Abbestr. 2-12, 10587 Berlin, Germany. E-mail: andrea.hornemann@ptb.de; Fax: +49 303481 7103; Tel: +493034817183

${ }^{b}$ Elettra-Sincrotrone Trieste S.C.p.A. di interesse nazionale, Strada Statale 14, 34149 Trieste, Italy

${ }^{c}$ BAM Bundesanstalt für Materialforschung und-prüfung, Richard-Willstätter-Str.10, 12489 Berlin, Germany

$\dagger$ Electronic supplementary information (ESI) available. See DOI: 10.1039/ c4cp05944a
}

pharmaceuticals, antigens, hormones, or toxins in a complex sample. However, there is usually a long way to go for the establishment of a bio-assay setup, which necessitates preliminary steps such as (i) the development of performance-based methods, (ii) the analysis of biological assays and (iii) their validation. This calls for advanced methodologies and readout procedures. Surface-Enhanced InfraRed Absorption (SEIRA) in combination with statistical tools addresses the challenges of accuracy, specificity, precision, and robustness by providing highly resolved vibrational information of complex molecular compounds. Surface-Enhanced InfraRed Absorption (SEIRA) constitutes an ideal platform to isolate vibrational spectroscopic signatures of targeted and active molecules (e.g. antibodies) of the bio-assay, and challenges the Surface-Enhanced Raman Spectroscopy (SERS) readout. ${ }^{1,2}$ Dissimilarly to SERS providing sensitive access to Raman-active modes in molecules, SEIRA allows for efficient detection of IR-active modes, thus providing complementary speciation information. Enhancement processes (see comparison in the ESI $\dagger$ ) of chemisorption to physisorption, for SERS and SEIRA, can modify the similarity of the effective speciation sensitivities of both methods. ${ }^{3-5}$

The present work addresses one of the design steps of an assay platform - the targeted biolabels consisting, in our case, of fluorophore-labeled antibodies chemisorbed onto gold nanoaggregates (Au NA), acting as nano-antennas for biocompatible, low-cost, amplified SEIRA detection ${ }^{6}$ and efficient fast discrimination for appropriate read-out in tunable and pre-selectable spectral ranges. ${ }^{3}$ 
Systematic analyses were performed on the model antibody Goat Anti-Mouse IgG and Goat Anti-Mouse IgG conjugated with fluorophores such as fluorescein isothiocyanate (FITC), rhodamine B isothiocyanate (RITC) and sulphorhodamine B dye (SULFO) fluorophores. These biolabels adsorbed at/nearby NP-functionalised (silanized) surfaces present additional and enhanced spectral signatures due to plasmonic modifications that were compared with those delivered from non-chemisorbed gold NA films simply incubated with the label components.

Extensive areas of dried sample films were analyzed by IR/SEIRA spectroscopy and the resulting complex hyperspectral datasets were submitted to automated statistical analysis, namely, principal component analysis (PCA). The relationship and dependency between chemical functional groups of various antibodyfluorophore conjugation systems were determined. Consequently, this paper presents profound insights into multivariate discrimination capabilities by direct visualization of derived structural clusters among various biolabel configurations.

\section{Material and methods}

For further details, please confer to the ESI. $\dagger$

\subsection{Synthesis of Au NP SEIRA substrates and immobilization of Au NPs on a MirrIR low-e-substrate}

$\mathrm{Au}$ NPs were synthesized according to a modified protocol of Lee and Meisel, ${ }^{7}$ yielding an estimated concentration of $1 \times$ $10^{-9} \mathrm{M}\left(\mathrm{ca} .7 \times 10^{14} \mathrm{NP} / \mathrm{L}\right)$ and characterized by UV/Vis-NIR spectroscopy (see Fig. S-1, ESI $\dagger$ ). For the preparation of SEIRA substrates, the immobilization of $\mathrm{Au}$ NPs was performed according to the protocol illustrated in ref. 8, using mid-infrared (MIR) low-e-slides.

\subsection{Synthesis of fluorophore-labeled antibody conjugates}

Different fluorescent dyes that are widely used for biomedical applications were studied. The conjugation of fluorophoreantibody was performed by a covalent linkage between the free residual amino groups of the antibody ${ }^{9}$ and the thiocyanate and sulfonyl groups of the fluorophores, ${ }^{10}$ and further characterized by MALDI-TOF-MS (Fig. S-2, ESI $\dagger$ ).

\subsection{Atomic force microscopy}

$\mathrm{Au}$ NP modified low-e-slides were studied using a NeaSNOM (Neaspec GmbH, Germany) in AFM mode for accessing the surface topography of the functionalized substrates and the existence of immobilized Au NPs. Sample areas of $8 \mu \mathrm{m} \times 8 \mu \mathrm{m}$ and $4 \mu \mathrm{m} \times 4 \mu \mathrm{m}$ were scanned with a corresponding pixel number of 400 pixels referring to a resolution of $20 \mathrm{~nm}(8 \mu \mathrm{m}$ scans) and 300 pixels referring to about $13 \mathrm{~nm}$ resolution (4 $\mu \mathrm{m}$-scans). A platinum iridium $\mathrm{V}$ coated monolithic silicon AFM tip with a tip radius of curvature with less than $25 \mathrm{~nm}$ (NanoWorld AG, Swiss) was used for probing the substrate surface. Image processing (median alignment, pixel column correction) was performed using Gwyddion 2.2.6 (CMI, Czech Republic). The surface topography depicts Au NA and a single
NP to be immobilized on the glass slide. The Au NPs showed spherical morphologies and were in the size range of about $40 \pm 3 \mathrm{~nm}$.

\subsection{SR-based FTIR micro-spectroscopy}

2.4.1 Instrument and data acquisition. The measurements were carried out on the IR beamline at the Metrology Light Source (MLS) storage ring using a Vertex-80v FTIR spectrometer ${ }^{11}$ coupled to an IR microscope Hyperion 3000 (Bruker Optics GmbH, Germany) equipped with a $128 \times 128$ pixels FPA (Focal Plane Array) detector (pixel size: $\sim 3 \mu \mathrm{m}$ at $15 \times$ magnification).

For FTIR micro-spectroscopy measurements, the SR source ( $\sigma_{x}=670 \mu \mathrm{m}, \sigma_{y}=183 \mu \mathrm{m}$, beam current $\sim 170 \mathrm{~mA}$ ) was focused through an IR cassegrain objective of 15 -fold magnification onto the sample films. MIR-spectra (from $3900 \mathrm{~cm}^{-1}$ to $900 \mathrm{~cm}^{-1}$ ) were acquired in transflection mode by co-addition of 128 scans at $16 \mathrm{~cm}^{-1}$ resolution. Background scans were collected before each sample measurement from a region free of sample and rationed against the sample spectrum. The use of synchrotron light has many advantages, thanks to its high brilliance, non-invasiveness, coherence and chemical sensitivity compared to conventional IR sources $^{12}$ and this study illustrates its applicability to the acquisition of large spectral datasets with FPA.

2.4.2 Sample preparation. For the biolabels, fluorophores and antibody-fluorophore conjugates, $2 \mu \mathrm{l}$ of the fluorophoreantibody conjugate solution was added to $20 \mu \mathrm{l}$ of suspended Au NPs, resulting in a final concentration of ca. $0.1 \mathrm{mg} \mathrm{ml}^{-1}$. After an incubation time of five minutes, the conjugate solution was drop-coated onto a low-e-slide, then dried in a desiccator. For comparison, the same amount of conjugate solution without NPs was drop-coated. The NPs $(20 \mu \mathrm{l})$ were exposed to $2 \mu \mathrm{l}$ of $10^{-4} \mathrm{M}$ FITC, RITC and SULFO solutions, respectively, resulting in a final concentration of $10^{-5} \mathrm{M}$. Fluorophores were prepared in DMF and further diluted in sodium bicarbonate buffer $(0.1 \mathrm{M}, \mathrm{pH} \sim 9)$ to a final concentration of $10^{-5} \mathrm{M}$. The antigen Mouse Anti-Goat IgG was pre-dissolved at a concentration of $0.5 \mathrm{mg} \mathrm{ml}^{-1}$ in borate buffered saline $(100 \mathrm{mM}, \mathrm{pH} \sim 8.2)$ and mixed with the colloidal suspensions to a final concentration of $0.05 \mathrm{mg} \mathrm{ml}^{-1}$. For Au NA-based SEIRA substrates, FTIR spectra were acquired in absorption mode in the $22000-900 \mathrm{~cm}^{-1}$ spectral range using a MCT (Mercury Cadmium Telluride) detector and an InGaAs photodiode $\left(16 \mathrm{~cm}^{-1}\right.$ spectral resolution, 128 scans). As a reference for immobilized Au NPs, a slide coated with APTMS for each discrete concentration was measured.

2.4.3 Data analysis: uni- and multivariate analyses. Each FPA measurement dataset comprised 16384 spectra, and due to the limited area illuminated by the bright SR beam the spectra contained regions with no MIR signal allowing for correcting the pixel noise and therefore enhancing the signal to noise ratios. The datasets for each of the 8 sample conditions (Goat Anti-Mouse IgG, Goat Anti-Mouse IgG with Au NA, fluorophore, fluorophore with $\mathrm{Au}$ NA, fluorophore conjugate, fluorophore conjugate with Au NA, Mouse IgG antibody with/without Au NA) were reduced to matrices of 550 spectra and loaded into Origin 8G (OriginLab Corporation, USA) for polynomial baselinecorrection. Each of these spectral dataset is issued from a 
sample region of $c a .37 \mu \mathrm{m} \times 96 \mu \mathrm{m}$, which is adequate for evaluating the potential of a FPA FTIR set-up as bioassay direct readout configuration. Indeed, the dimension of the surface probed here corresponds to bio-diagnostically relevant areas delivered by sample systems spotted onto microarrays which range typically from $10 \mu \mathrm{m}$ up to $500 \mu \mathrm{m} .^{13}$

Principal component analysis (PCA): the second derivatives of the full dataset of 4400 baseline-corrected absorbance spectra were calculated and consecutively submitted to the SavitzkyGolay algorithm (5 smoothing points) and vector-normalization before PCA using MatlabR2011a (The Mathworks Inc., Natick, USA) and PLS toolbox (Eigenvector Research, Inc., USA). The principle components (PCs) were calculated from PC1 up to PC4 and illustrated as scatter plots; such a high-order approach has been needed for discriminating certain label components.

\section{Results and discussion}

\subsection{Au NA-immobilization on MirrIR low-e-MIR substrates for SEIRA studies}

Self-assembled Au NP multilayers have successfully been employed as versatile platforms for molecular sensing approaches. ${ }^{14}$ Fig. 1 displays the spectral features of $\mathrm{Au}$ nanoparticulate multilayers that were generated during the drop-coating procedure and analysed in the $22000-900 \mathrm{~cm}^{-1}$ region. Due to aggregation of the Au NPs, a red-shift of the plasmon band is induced, and a broader plasmon band appears, extending from the NIR to the MIR region (compare with Fig. S-1, ESI $\dagger$ ). The position of the extended plasmon band shows that pre-resonant excitation by surface plasmons in the MIR region can be exploited for the SEIRA studies. This is in accordance with previous observations, ${ }^{15}$ where a continuum-like resonance for Au nanoshell array samples was observed in the MIR spectral range.

\subsection{MIR signatures delivered by fluorophore-antibody NP biolabels}

Similar to clinical diagnostics, dried films of serum proteins or antibodies are convenient for FTIR investigations. The study of the secondary structure composition, structural dynamics and/or the study of conformational changes (effects of ligand binding, temperature, and $\mathrm{pH}$ ) of the antibodies in the liquid phase is not the aim of this work presented here. In the following we discuss the signatures from SEIRA NP-labeled

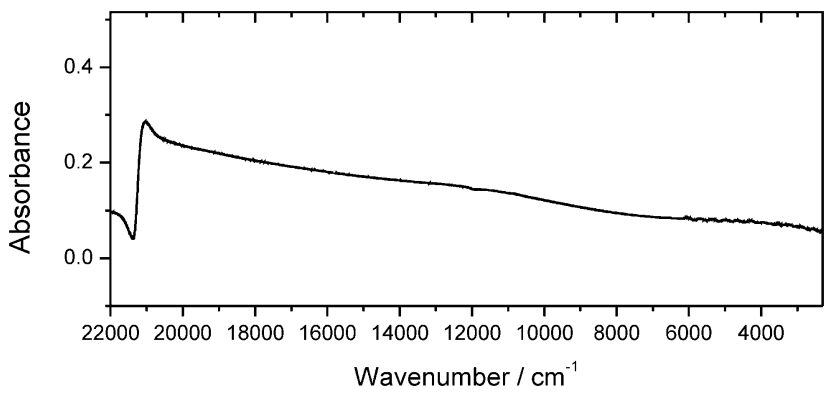

Fig. 1 Absorbance spectrum of Au NA showing an extended plasmon band in the mid-infrared region used for the SEIRA experiments. Analysis was performed in the spectral range from $22000 \mathrm{~cm}^{-1}$ to $900 \mathrm{~cm}^{-1}$. biolabels (such as antibodies and conjugates) that can be used to visualize and ultimately quantify the bound analyte which is not that obvious or detected in the conventional FTIR configuration. Of notice that it is inherent to the SEIRA technique compared to conventional FTIR is the influence of the metal surface that may lead to some spectral shapes, shifts and changes in intensities. ${ }^{16}$

3.2.1 Goat anti-mouse IgG-FITC conjugate. The spectral properties of unlabeled and labeled Goat Anti-Mouse antibodies (Fig. 2A and E) were studied. The signature of Goat Anti-Mouse IgG exhibits two strong IR bands at $1535 \mathrm{~cm}^{-1}$ and $1635 \mathrm{~cm}^{-1}$ that refer to the Amide II and Amide I bands characteristic of proteins $^{17}$ (Table S-1, ESI $\dagger$ ). In the relatively large wavenumber region $\left(3741 \mathrm{~cm}^{-1}\right.$ to $3278 \mathrm{~cm}^{-1}$ ) the band intensity of the stretching (str.) vibrations corresponding to $-\mathrm{NH}$ and $-\mathrm{OH}$

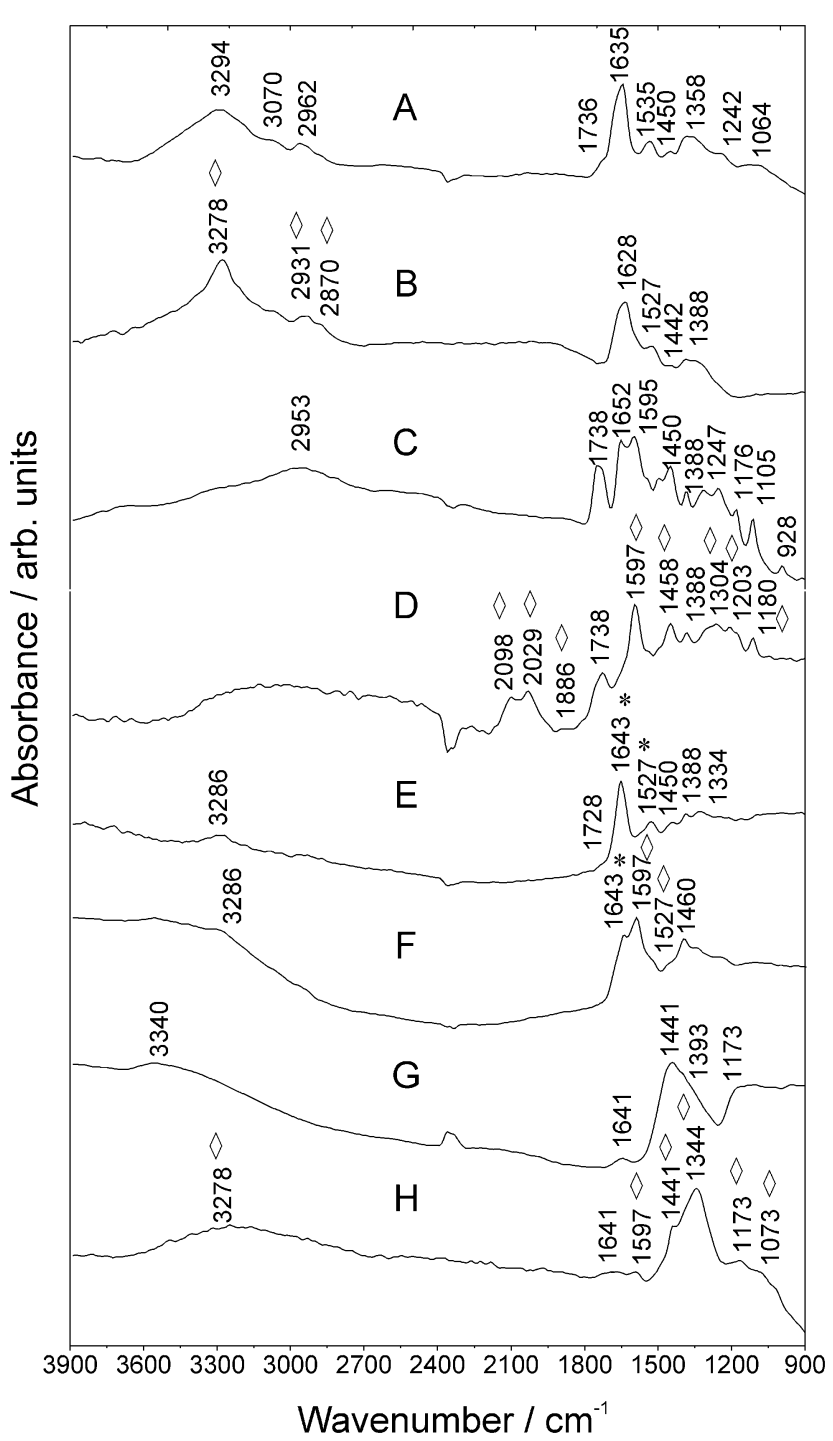

Fig. 2 MIR spectra of (a) Goat Anti-Mouse IgG, (b) Au NA incubated with Goat Anti-Mouse IgG, (c) FITC, (d) Au NPs and FITC, (e) Goat Anti-Mouse IgG-FITC, (f) Au NPs and Goat Anti-Mouse lgG-FITC and the antigen (g) Mouse Anti-Goat lgG and (h) Au NPs and Mouse Anti-Goat lgG measured using SR. Vibrations of FITC are marked with asterisks *. Enhanced vibrations are marked with rhombohedra $\diamond$. For further details, refer to Table S-1 (ESI $)$ ). 
groups of amino acid residues ${ }^{18}$ which form the secondary structure of the antibody is enhanced in the presence of Au NPs (see rhombohedra $\diamond$ ). This observation corresponds to IR bands that were detected from other protein-Au bioconjugates. ${ }^{19}$ The spectrum delivered by Goat Anti-Mouse IgG incubated with Au NPS (Fig. 2B) shows a similar spectral pattern as displayed in Fig. 2A. The band at $c a .3278 \mathrm{~cm}^{-1}$ is attributed to $-\mathrm{NH}$ str. vibrations of antibody proteins. ${ }^{20}$ The signature of FITC is presented in Fig. $2 \mathrm{C}$ and exhibits bands referring to str. vibrations (region from $1652 \mathrm{~cm}^{-1}$ to $1496 \mathrm{~cm}^{-1}$ ) of the xanthene ring moiety and external group modes. ${ }^{21}$ The MIR signature delivered by FITC molecules adsorbed onto the Au NPs is shown in Fig. 2D and comprises str. vibrations of the carbonyl groups of FITC ${ }^{21}$ (see asterisks ${ }^{*}$ ). The newly occurring band originates from $-\mathrm{C}=\mathrm{N}$ and $-\mathrm{C}=\mathrm{C}$ str. vibrations. This can be explained by different binding orientations which are restricted due to the fluorophore-NP incubation procedure during which the chemisorbed FITC molecules tend to orient. In addition, the origin of SEIRA being observed for Au NP-FITC is due to the formation of large colloidal $\mathrm{Au}$ NA, presumably induced by the drop-coating process. The NA chemisorbed FITC molecules orient their dipole moments to be in line with the local electric field of the metal NA, resulting in much larger absorption than when they are not in line with the oscillating electric field. ${ }^{22,23}$ Such larger absorption of FITC molecules can be exploited for building complex conjugates for sensitive multiplexed assay approaches.

An enhancement of the modes in the spectral region between $1304 \mathrm{~cm}^{-1}$ and $1180 \mathrm{~cm}^{-1}$, as well as at $c a .1458 \mathrm{~cm}^{-1}$ can be observed as well (compare Fig. 2C and D). The Goat Anti-Mouse IgG-FITC signature in Fig. 2E comprises lines which can be assigned to typical bands of FITC (see asterisks *) and bands between $3720 \mathrm{~cm}^{-1}$ and $1103 \mathrm{~cm}^{-1}$ referring to antibody specific signatures. Fig. 2F shows the SEIRA spectrum of the antibody-FITC conjugate recorded in the presence of Au NA. This signature comprises lines which can be assigned to FITC typical bands (Table S-1, ESI $\dagger$ ). For example, in the vicinity of the mode at ca. $1643 \mathrm{~cm}^{-1}$ (str. vibrations of the xanthene skeleton) of the unenhanced antibody-FITC conjugate now a broad shoulder being located at $1597 \mathrm{~cm}^{-1}$ appears in the SEIRA spectrum of the antibody-FITC conjugate. This vibration originates from the aromatic $-\mathrm{C}-\mathrm{C}$ str. vibrations of the xanthene ring skeleton of FITC molecules which was already observed in the SEIRA spectrum of FITC (compare Fig. 2D). This shows that SEIRA is able to detect with high sensitivity fluorophore molecules in their conjugated states along with their molecular orientation.

Considering the intended use of labeled antibodies in immunoassays, the signatures of the targeting counterpart Mouse Anti-Goat IgG were studied. Fig. 2G illustrates antigen specific lines being typical for antibody protein vibrations. The SEIRA signature of the Mouse IgG antibody is displayed in Fig. $2 \mathrm{H}$ that shows enhanced modes in the spectral region between $1800 \mathrm{~cm}^{-1}$ and $900 \mathrm{~cm}^{-1}$. Another possible contribution which may explain the observed spectral differences is due to chemical enhancement. Molecules strongly chemisorbed onto a (noble) metal surface show a larger enhancement than do physisorbed molecules, suggesting a chemical effect between the molecule and the surface due to strong interactions (Au NA-antibody complexation). This mechanism would describe the case that we discuss and correspond to our antibodies that we detect in the presence of Au NA. It is known that the absorption coefficients of chemisorbed molecules are larger than those of condensed overlayers and therefore interactions between adsorbed molecules may also affect the intensity and thus impact and explain the enhancement of some modes. Charge oscillations between molecular orbitals and the metal surface may enlarge the absorption coefficients of adsorbates. ${ }^{2,24}$

3.2.2 Goat anti-mouse IgG-RITC conjugate. In order to study the multiplexing capability of further fluorophore conjugates, RITC, a rhodamine derivative, ${ }^{25}$ was used for the coupling. Fig. 3B and D display the MIR signatures of RITC and the spectrum of Goat Anti-Mouse IgG-RITC conjugate recorded in the presence of Au NA. Since both fluorophores FITC and RITC have quite similar molecular composition their MIR signatures exhibit the same modes, i.e., the bands at $1172 \mathrm{~cm}^{-1}$ $(\delta \mathrm{C}-\mathrm{OH})$ and at $c a .2941 \mathrm{~cm}^{-1}\left(\nu_{\mathrm{as}} \mathrm{CH}_{2}\right.$ vibration $)$ are observed in both MIR fingerprints. ${ }^{21}$ However, some spectral differences are noticeable. Compared to the spectrum of FITC, the main peak in the RITC spectrum is located at $1589 \mathrm{~cm}^{-1}$ (Fig. 3A) which can be attributed to aromatic str. vibrations. The enhanced intensities of the vibrational modes referring to RITC are highlighted with rhombohedra $\diamond$. The mode at $1743 \mathrm{~cm}^{-1}$ can be assigned to a $-\mathrm{C}(=\mathrm{O}) \mathrm{H}$ str. vibration. ${ }^{26}$ The SEIRA spectrum of RITC recorded on Au NA (Fig. 3B) exhibits an enhanced mode at $1589 \mathrm{~cm}^{-1}$. It is remarkable that the relative intensity of the str. mode of the xanthene ring moiety at $1342 \mathrm{~cm}^{-1}$ experiences an enhancement

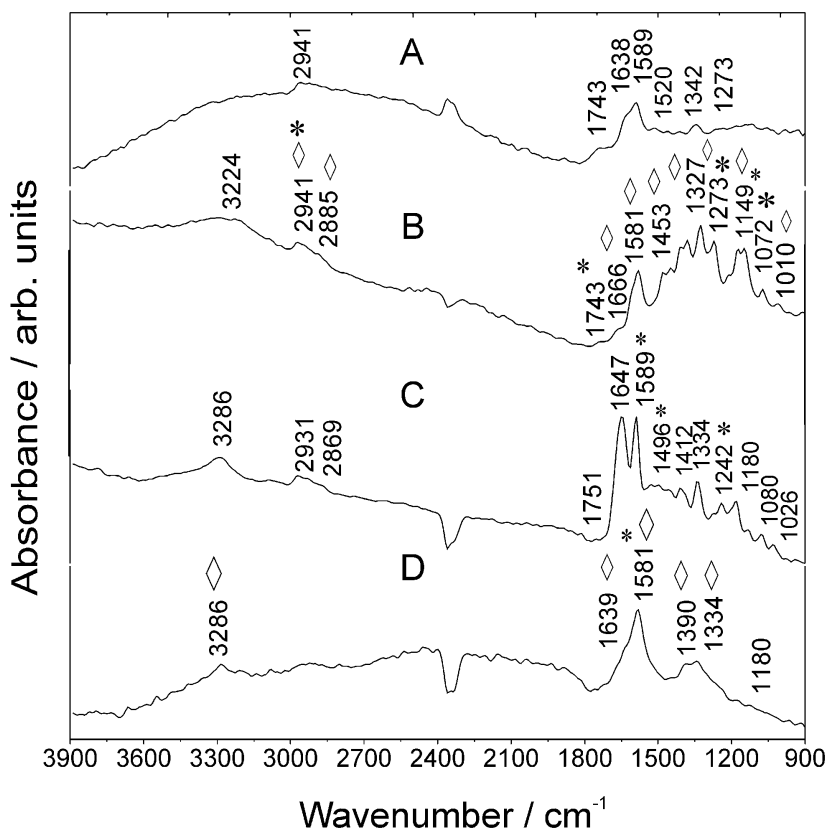

Fig. 3 MIR spectra of (a) RITC, (b) Au NPs and RITC, (c) Goat Anti-Mouse IgG-RITC, and (d) Au NPs and Goat Anti-Mouse IgG-RITC measured using SR. Vibrations of RITC are marked with asterisks *. Enhanced vibrations are marked with rhombohedra $\diamond$. For further details, refer to Table S-2 (ESI†). 
by a factor of five, which proves again the selectivity of revealing one of the most specific molecular groups of the fluorescent dye.

In the case of RITC measured on Au NA, further new IR vibrations are observed, for instance, at $1666 \mathrm{~cm}^{-1}$. Further, the mode at $1520 \mathrm{~cm}^{-1}$ disappears which is caused by a change in the molecular orientation. The spectrum of the antibody-RITC conjugate (Fig. 3C) exhibits RITC-typical modes which are also observed in the bare fluorophore spectrum (Fig. 3A). For instance, the mode at $1589 \mathrm{~cm}^{-1}$ originates from aromatic $-\mathrm{C}-\mathrm{C}$ str. vibrations of RITC molecules. $^{26}$ In the presence of $\mathrm{Au} \mathrm{NA}$, the conjugate signature (Fig. 3B) delivers one broad band at $1589 \mathrm{~cm}^{-1}$ and the mode at $1647 \mathrm{~cm}^{-1}$ is observed as a shoulder being located at $c a$. $1639 \mathrm{~cm}^{-1}$ (Table S-2, ESI $\dagger$ ).

3.2.3 Goat anti-mouse IgG-SULFO conjugate. Fig. $4 \mathrm{~B}$ and D shows the signatures of the SULFO and SULFO-labeled Goat Anti-Mouse antibody, in the presence of $\mathrm{Au}$ NA. Compared to the unenhanced signature of SULFO (Fig. 4A), the mode referring to the aromatic $-\mathrm{C}-\mathrm{C}$ str. vibrations ${ }^{27}$ at $1581 \mathrm{~cm}^{-1}$ is enhanced, as the line at $1597 \mathrm{~cm}^{-1}$ is shifted to shorter frequencies. Further modes originate from $-\mathrm{C}-\mathrm{O}$ str. vibrations of the carbonyl group and $-\mathrm{C}-\mathrm{C}$ str. vibrations nearby the xanthene ring moiety. ${ }^{28}$ The SEIRA signature of SULFO (Fig. 4B) shows structural differences in terms of band shifts and its spectral behaviour in the proximity of Au NA is quite similar to FITC (compare Fig. 2D). Further, new bands appear in the long frequency region, for example, lines at $3186 \mathrm{~cm}^{-1}$ referring to $-\mathrm{NH}$ str. vibrations at $3325 \mathrm{~cm}^{-1}$ can be observed along with str. vibrations at $3780 \mathrm{~cm}^{-1}(-\mathrm{OH}){ }^{29}$ In the spectrum of the antibody-SULFO conjugate the band at $1597 \mathrm{~cm}^{-1}$ is red-shifted. However, conjugate typical bands appear at

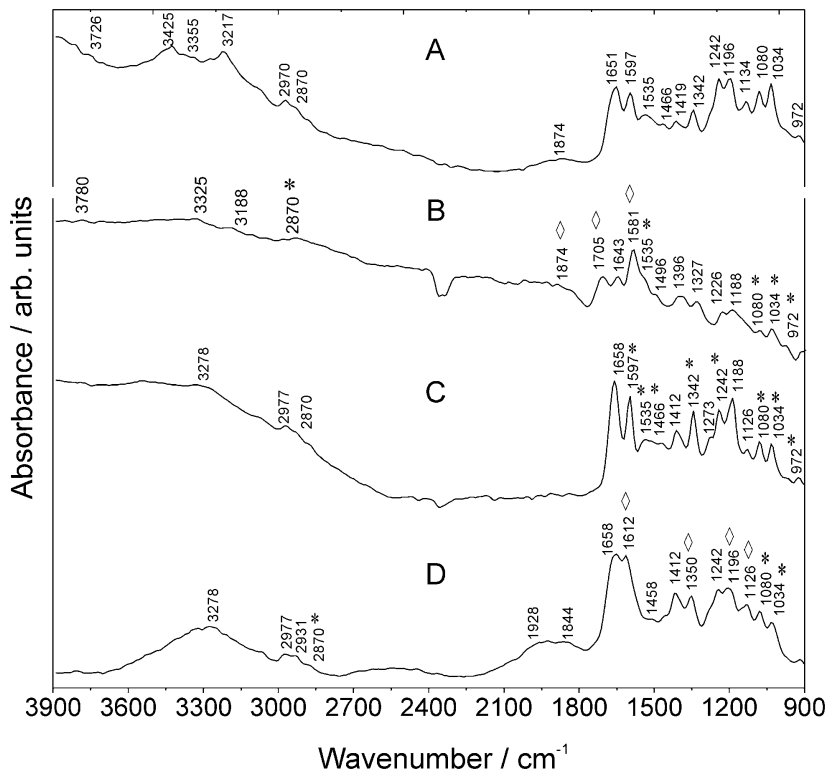

Fig. 4 MIR spectra of (a) SULFO, (b) Au NPs and SULFO, (c) Goat AntiMouse IgG-SULFO, and (d) Au NPs and Goat Anti-Mouse IgG-SULFO measured using SR. Vibrations of SULFO are marked with asterisks * Enhanced vibrations are marked with rhombohedra $\diamond$. For further details, refer to Table S-3 (ESI†).
$1412 \mathrm{~cm}^{-1}$ along with modes at $1034 \mathrm{~cm}^{-1}$ and $1242 \mathrm{~cm}^{-1}$ originating from SULFO. The spectrum of the antibody-SULFO conjugate recorded in the presence of $\mathrm{Au}$ is very similar to the conjugate spectra recorded without Au NA (refer to Table S-3, ESI $\dagger$ ).

The univariate study of these different antibody-fluorophore conjugate signatures shows that the electric field enhancing capabilities differ between Au NPs and the type of antibodyfluorophore conjugate which justifies the systematic approach on the spectral properties of such fluorophore-antibody NP complexes. Additionally, this shows that for the bioassay design, SEIRA induced by NPs provides different vibrational information from fluorophore-anibody NP labels that can be used for building up spectral databases.

Summarizing the results, FTIR spectroscopy combined with SEIRA is an important analytical tool for studying the biochemical composition of proteins such as antibodies. Bands of high interest for biological purposes and labeling procedures can be found in the Amide I/II and ester regions. The Amide I region is traditionally exploited for probing the secondary structures of proteins, as an indicator band for monitoring spectral changes due to alterations in the molecular structure, usually due to external perturbations. Our studies show that complex biolabels that are read out by SEIRA deliver distinct spectra that clearly differ from their unenhanced counterparts, the conventional FTIR spectra (please refer to Fig. S-3, and Tables S-1-S-3, ESI $\dagger$ ).

\subsection{Multivariate discrimination of the signatures of label components}

PCA as a powerful chemometric method for finding patterns in complex FTIR datasets enables fast identification and separation of labeled antibodies which are designed for multianalyte detection. $^{30,31}$ Fig. 5 shows score plots (PC1-PC4) that entail spectral differences. All PCA procedures displayed in Fig. 5A-C (and Fig. 6b) were performed by repetitive experiments (data not shown here, 95\% reproducibility).

The PCA displays datasets of the Goat Anti-Mouse IgG that are well separated from the antigen data in PC1 against PC2. The results of PCA confirm that on average $85 \%$ of the variance of the spectral datasets, i.e. the discrimination capability up to the third order, could be explained for the fluorophores. Additionally, we decided to put on display all components up to the fourth order to illustrate the options for discriminating different label and/or label components. The scores (PC1 against PC2) of Goat Anti-Mouse IgG are positive and lie in the second quadrant of the variance-weighted room. In PC1 against PC3, the Goat Anti-Mouse IgG antibody data are well separated from the unenhanced spectra. The spectral differences observed in the SEIRA spectrum of FITC (see Fig. 2D) are also reflected by PC1 against PC2. Fig. 5A illustrates that in PC1 against PC2 FITC is separated from the FITC-labeled antibody. With respect to the design of multiplexed immunoassay formats, this shows that the enhancement capabilities of different Au NA labels provide MIR signatures with different spectral patterns. PCA for studying the multiplexing capability of the other fluorophore conjugates containing RITC molecules was 

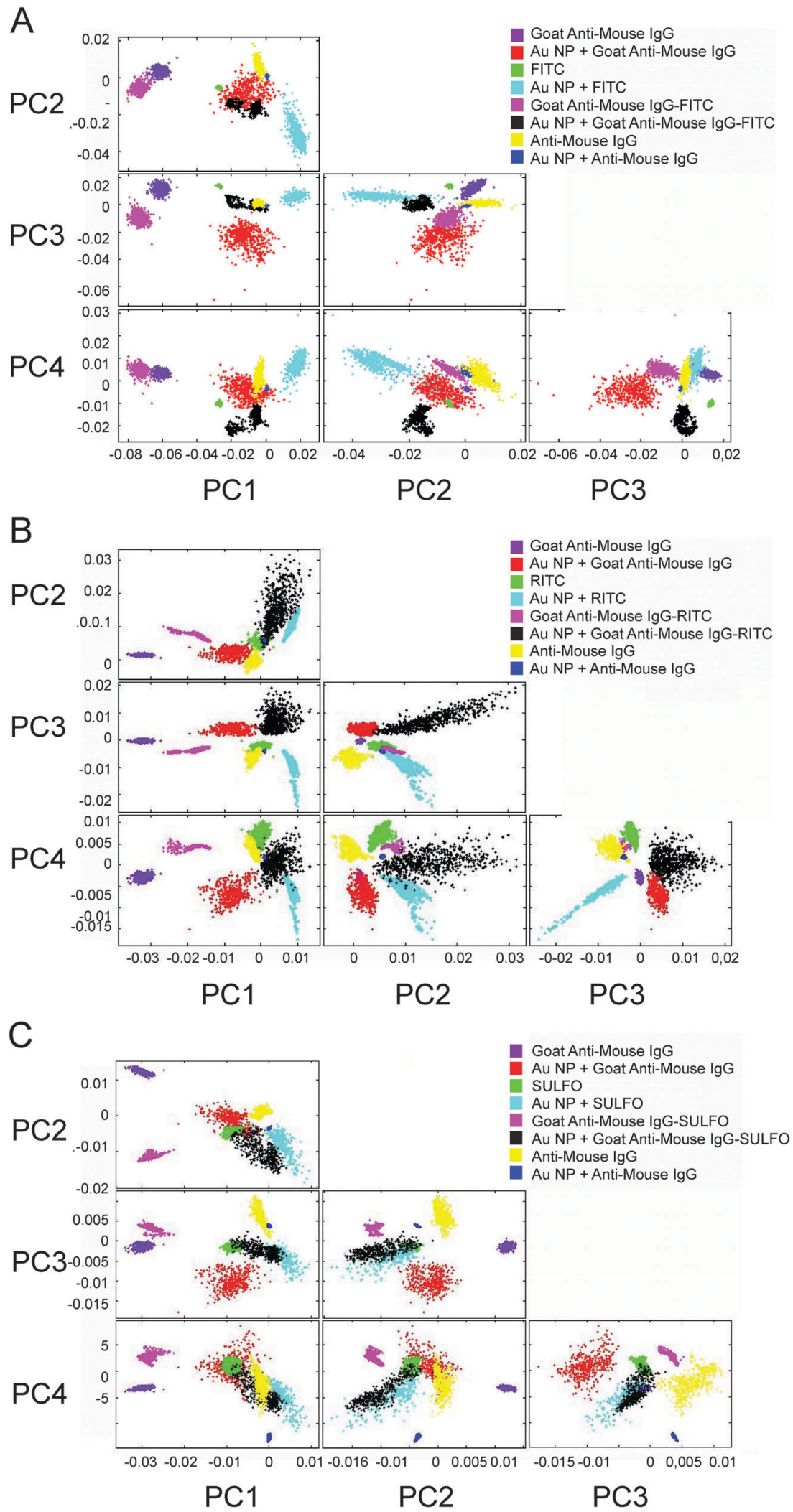

Fig. 5 PCA illustrated from PC1 to PC4. PCA was performed with vector-normalized second derivative spectra over the spectral range from $3900 \mathrm{~cm}^{-1}$ to $900 \mathrm{~cm}^{-1}$. PCA clusters are color-coded as follows: Goat Anti-Mouse lgG (violet), Au NPs and Goat Anti-Mouse IgG (red), fluorophore (green), Au NPs and fluorophore (cyan), Goat Anti-Mouse IgG-fluorophore (magenta), Au NP and Goat Anti-Mouse IgG-fluorophore (black), Mouse Anti-Goat IgG and NPs (yellow) and Mouse Anti-Goat IgG with Au NPs (blue). 
also performed successfully. Similar to the PC scores illustrated in Fig. 5A, a discrimination of the RITC MIR signatures measured on Au NA in PC1 against PC2 was feasible. A variance of about $80 \%$ is captured by the components PC1-PC3. The Goat Anti-Mouse IgG-RITC conjugate cluster has quite similar distance towards its unlabeled counterpart as the FITC-antibody conjugate, which is reflected by the good separation in PC1 against PC2.

Compared to all other fluorophore-antibody conjugates discussed so far, the SEIRA signature of the FITC-labeled Goat Anti-Mouse IgG is most similar to the unlabeled antibody sample; for SULFO the distance in PC1 against PC2 is the highest which shows the increase of discrimination capability of the conjugated fluorophores.

The multivariate analysis of spectral features is an important scope for the design and development of bioassay platforms, as functionalization with biolabel components may require several preparation steps before the establishment of a functionally completed assay setup.

\subsection{MIR signatures of the antibody-FITC conjugate measured on the Au NP modified low-e-substrate}

Fig. 6a shows the extinction spectrum and surface topography of $\mathrm{Au}$ NPs being immobilized on an APTMS-functionalized substrate (1\% APTMS, time of incubation: 24 h, see Fig. 6a, A-C). A second extended plasmon band is observed in the MIR region and proves the existence of $\mathrm{Au} \mathrm{NA}^{32}$ as for the unmodified glass substrate. This is important in the case of the enhancement capabilities of immobilized NPs as large NA reaching microscale size dimensions of bulk materials might drastically lose their enhancement properties.

The PCA results confirm that on average $85 \%$ of the variance of the spectral datasets up to the third order could be explained. PCA of the Mouse Anti-Goat IgG signatures shows (a)

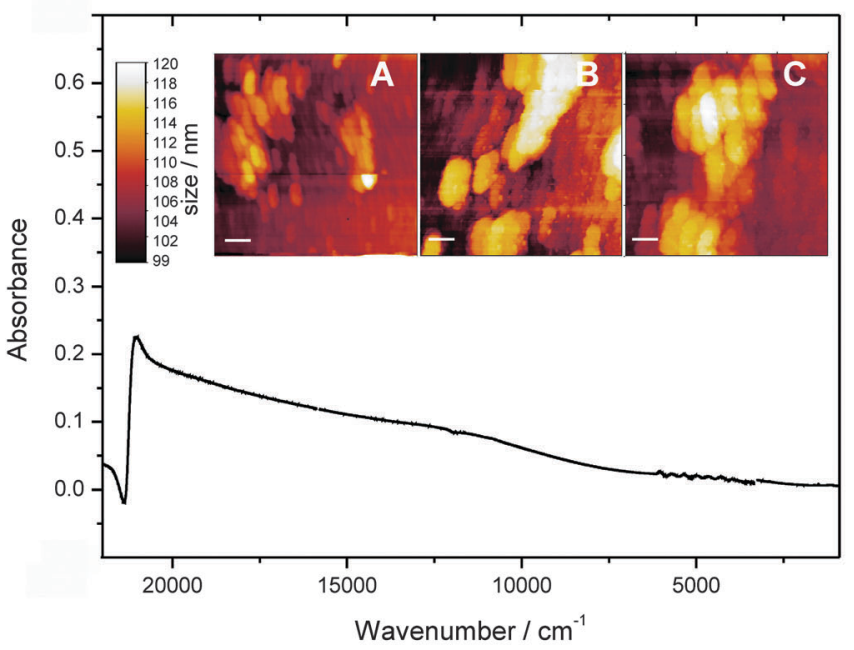

(b)

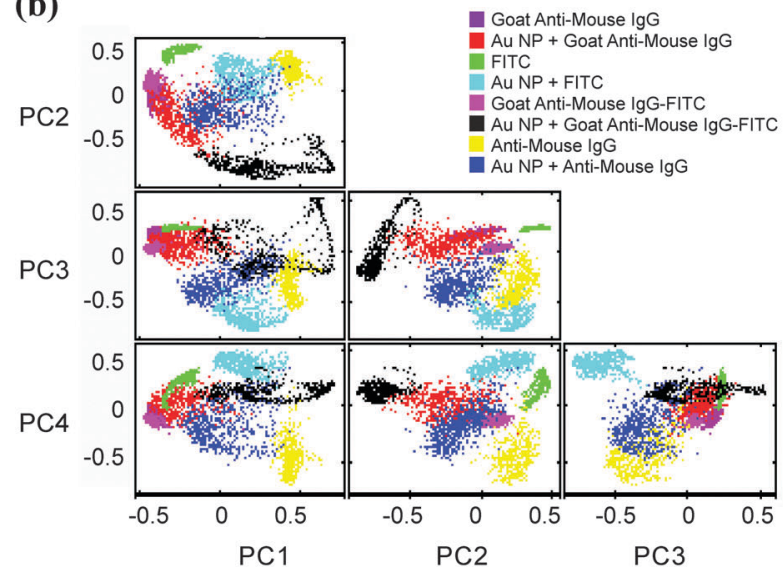

(c)

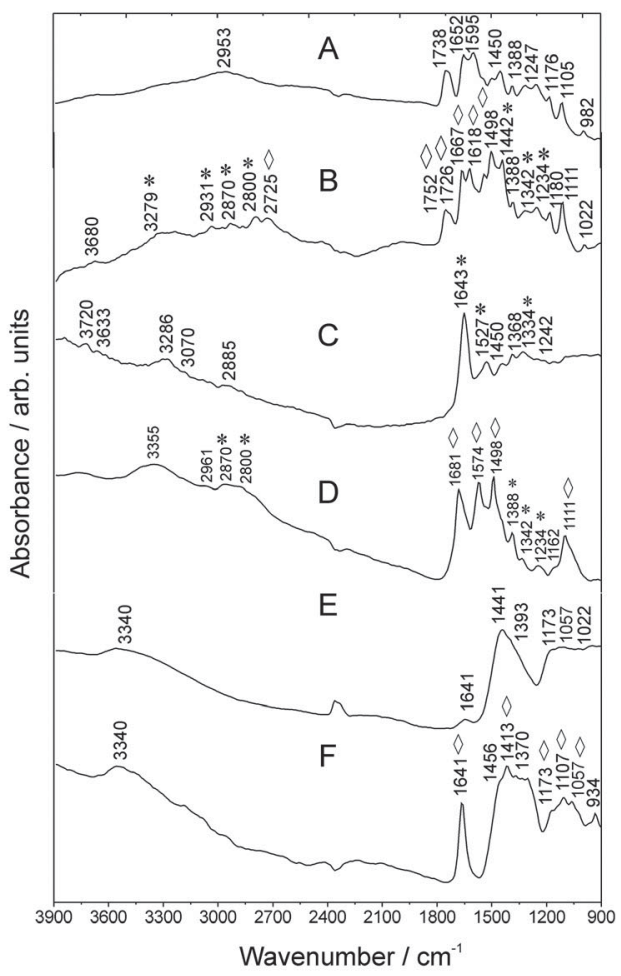

Fig. 6 (a) Absorbance spectrum of Au NPs immobilized on an APTMS-functionalized substrate (CAPTMS 1\%, incubation time: 2 h) and the corresponding surface topography (scale bars for (a): $0.1 \mu \mathrm{m}$, (b) and (c): $0.05 \mu \mathrm{m}$ ). Au NA are visible as spherical shaped clusters. (b) PCA analysis (3900-900 $\mathrm{cm}^{-1}$ ). (c) MIR signatures of (a) FITC, (b) FITC on the APTMS-substrate, (c) Goat Anti-Mouse IgG-FITC and (d) Goat Anti-Mouse IgG-FITC on the APTMS-substrate and the antigen (e) Mouse Anti-Goat IgG and (f) Mouse Anti-Goat IgG on the NP-modified APTMS substrate measured using SR. Vibrations of FITC are marked with asterisks *. Enhanced vibrations are marked with rhombohedra $\diamond$. 
a better separation from the enhanced ones (PC1 against PC2 and PC3 in Fig. 6b). The MIR signatures are illustrated in Fig. 6c. Compared to the FITC signature, spectral differences in the enhanced counterpart can be observed, i.e., the band at $1738 \mathrm{~cm}^{-1}$ (compare with Fig. 3) is split into lines at $1726 \mathrm{~cm}^{-1}$ and $1752 \mathrm{~cm}^{-1}$. The dependence on the substrate configuration and modification is very striking when comparing the antibody-FITC conjugate being adsorbed onto the Au NA on the APTMS-modified slide - the signature exhibits FITC typical bands along with antibody modes (Fig. 6c, C). Compared to the conjugate signature in Fig. 2 where a mode at $c a .1643 \mathrm{~cm}^{-1}$ can be observed, now a new band at $1681 \mathrm{~cm}^{-1}(-\mathrm{C}=\mathrm{O}$ str. appears after APTMS modification. This shows that the SEIRA signature of FITC and its antibody conjugate differs from the signatures measured with the APMS-modified substrate and the assay configuration itself. It should be noted that the vibrational modes in the $1600 \mathrm{~cm}^{-1}$ to $1300 \mathrm{~cm}^{-1}$ range can be assigned to both aminopropyl and free amino groups as well. However, no peak at $1491 \mathrm{~cm}^{-1}$ associated with $\mathrm{NH}^{3+}$ in the $\mathrm{SiO}^{-}-\mathrm{H}-\mathrm{NH}^{3+}$ group of APTMS residues ${ }^{33}$ is noticeable which shows that this substrate enables the overlap-free detection of amino-group modes delivered by the targeting units themselves.

A tremendous impact of this substrate with respect to its enhancement capability is shown for the antigen Mouse AntiGoat (Fig. 6c, F) in the region from $1460 \mathrm{~cm}^{-1}$ to $1300 \mathrm{~cm}^{-1}$. In particular, the mode at $1641 \mathrm{~cm}^{-1}$ is extremely intensified by a factor of about 8. This is in accordance to the calculated dimension of the average enhancement factor for the MIR spectral region as described in ref. 34 .

\section{Conclusions}

We demonstrate the efficiency of the SEIRA technique to probe the molecular characteristics of different antibody-fluorophore NP conjugates considered in this study. Our measurements show that the spectral signatures of the native antibody Goat Anti-Mouse IgG and the conjugated fluorophores are preserved in the proximity of Au NPs but enhanced by the latter. This implies that the elaboration of specific assay platform spectral databases can be envisaged with SEIRA. Coupled with PCA analysis, this method allows clear discrimination of the functionalized systems, which ascertain their potential for multiplexed bioassay applications and biosensor development of high efficiency with customized responses. Our work highlights the prospective use of NP-assays for biodiagnostics ${ }^{35,36}$ and as catalysts for selectively enhancing bio-physiological responses of cells or bacteria. Consequently, SEIRA applications ${ }^{37}$ can be expected to show even higher performance characteristics than the conventional/established methods such as SERS. ${ }^{38}$

As such, a logical extension of our work is to confront the potentialities of SEIRA to discriminate biolabels' spectral features in a real biological environment, which is a subject of another communication.

Altogether, this paper evidences the availability of a novel means in the complex bio-diagnostic micro-assay toolbox to identify the suitability of biolabel systems along with their discrimination potential and their multiplexing capability. Enhancing functionality performances is the key point to obtain higher and targeted screening performance, the ultimate goal of complex bio-diagnostic assay platforms. ${ }^{39}$

\section{Acknowledgements}

We thank Ralf Bienert (BAM Federal Institute for Materials Research and Testing) for use of the DLS apparatus. The machine-physicists team of the Helmholtz-Zentrum Berlin (HZB) is gratefully acknowledged for tuning the MLS according to our experimental needs. We thank Arne Hoehl of the MLS team of the PTB for helpful discussions and use of the beamline instrumentation. Sincere thanks are given to Sören Selve (ZELMI, TU Berlin) for the preparation of the TEM images.

\section{References}

1 G. C. Schatz, THEOCHEM, 2001, 573, 73-80.

2 M. Osawa and K.-i. Ataka, Surf. Sci., 1992, 262, L118-L122.

3 S. Lal, S. Link and N. J. Halas, Nat. Photonics, 2007, 1, 641-648.

4 M. Osawa and M. Ikeda, J. Phys. Chem., 1991, 95, 9914-9919.

5 A. Hartstein, J. R. Kirtley and J. C. Tsang, Phys. Rev. Lett., 1980, 45, 201-204.

6 H. D. Wanzenböck, B. Mizaikoff, N. Weissenbacher and R. Kellner, Fresenius' J. Anal. Chem., 1998, 362, 15-20.

7 P. C. Lee and D. Meisel, J. Phys. Chem., 1982, 86, 3391-3395.

8 C. D. Geddes, H. Cao, I. Gryczynski, Z. Gryczynski, J. Fang and J. R. Lakowicz, J. Phys. Chem. A, 2003, 107, 3443-3449.

9 G. T. Hermanson, in Bioconjugate Techniques, ed. A. Press, Elsevier Inc., London, 2008, vol. 2, pp. 396-430.

10 H. Rinderknecht, Nature, 1962, 193, 167-168.

11 A. Gottwald, R. Klein, R. Müller, M. Richter, R. Thornagel and G. Ulm, Metrologia, 2012, 49, S146-S151.

12 P. Dumas, G. D. Sockalingum and J. Sulé-Suso, Trends Biotechnol., 2007, 25, 40-44.

13 M. Bally, M. Halter, J. Voros and H. M. Grandin, Surf. Interface Anal., 2006, 38, 1442-1458.

14 F. Toderas, S. Boca, M. Baia, L. Baia, D. Maniu, S. Astilean and S. Simon, J. Optoelectron. Adv. Mater., 2007, 9, 625-628.

15 F. Le, D. W. Brandl, Y. A. Urzhumov, H. Wang, J. Kundu, N. J. Halas, J. Aizpurua and P. Nordlander, ACS Nano, 2008, 2, 707-718.

16 G. Dovbeshko, O. Fesenko and A. Nazarova, J. Phys. Stud., 2006, 10, 127-134.

17 F. S. Parker and D. M. Kirschenbaum, Nature, 1960, 187, 386-388.

18 A. W. Herlinger and T. Veach Long, J. Am. Chem. Soc., 1970, 92, 6481-6486.

19 A. A. Kamnev, L. A. Dykman, P. A. Tarantilis and M. G. Polissiou, Biosci. Rep., 2002, 22, 541-547. 
20 F. Tian, R. Middaugh, T. Offerdahl, E. Munson, S. Sane and H. Rytting, Int. J. Pharm., 2007, 335, 20-31.

21 L. L. Wang, A. Roitberg, C. Meuse and A. K. Gaigalas, Spectrochim. Acta, Part A, 2001, 57, 1781-1791.

22 D. Ross and R. Aroca, J. Chem. Phys., 2002, 117, 8095-8103.

23 M. Osawa, K. Ataka, K. Yoshii and Y. Nishikawa, Appl. Spectrosc., 1993, 47, 1497-1502.

24 M. Osawa and S. Kawata, Surface-Enhanced Infrared Absorption, Springer, Berlin/Heidelberg, 2001, vol. 81, pp. 163-187.

25 P. Hildebrandt and M. Stockburger, J. Phys. Chem., 1984, 88, 5935-5944.

26 M. Majoube and M. Henry, Spectrochim. Acta, Part A, 1991, 47, 1459-1466.

27 Y.-F. Fang, Y.-P. Huang, D.-F. Liu, Y. Huang, W. Guo and D. Johnson, J. Environ. Sci., 2007, 19, 97-102.

28 R. Markuszewski and H. Diehl, Talanta, 1980, 27, 937-946. 29 T. Ohishi, J. Non-Cryst. Solids, 2003, 332, 80-86.

30 R. G. Brereton, Chemometrics: data analysis for the laboratory and chemical plant, John Wiley \& Sons Ltd, Chichester, UK, 2006, pp. 183-269.
31 C. A. Sellick, R. Hansen, R. M. Jarvis, A. R. Maqsood, G. M. Stephens, A. J. Dickson and R. Goodacre, Biotechnol. Bioeng., 2010, 106, 432-442.

32 A. M. Schwartzberg, C. D. Grant, A. Wolcott, C. E. Talley, T. R. Huser, R. Bogomolni and J. Z. Zhang, J. Phys. Chem. B, 2004, 108, 19191-19197.

33 Y. Zhang, N. Kohler and M. Zhang, Biomaterials, 2002, 23, 1553-1561.

34 R. Aroca, Surface-enhanced Vibrational Spectroscopy, John Wiley and Sons Ltd, West Sussex, 2007, p. 198.

35 Y. Ding, X. Chu, X. Hong, P. Zou and Y. Liu, Appl. Phys. Lett., 2012, 100, 013701.

36 A. Quershi, Y. Gurbuza, W. P. Kang and J. L. Davidson, Biosens. Bioelectron., 2009, 25, 877-882.

37 R. Adato and H. Altug, Nat. Commun., 2013, 4, 2154.

38 M. Kahraman, A. I. Zamaleeva, R. F. Fakhrullin and M. Culha, Anal. Bioanal. Chem., 1995, 395, 2559-2567.

39 P. Qiu, E. F. Simonds, S. C. Bendall, K. D. Gibbs Jr, R. V. Bruggner, M. D. Linderman, K. Sachs, G. P. Nolan and S. Plevritis, Nat. Biotechnol., 2011, 29, 886-891. 